multiple comparisons using Tukey's HSD test indicated that children in the high-income group had lower levels of motivation $(M=7.29$, $\mathrm{SD}=2.07)$ compared to children in the middle $(\mathrm{M}=8.18)$ and low $(\mathrm{M}=8.70)$ income groups. Level of motivation did not differ for children in the middle and low-income groups. Finally, parent motivation level did not differ significantly by income group. While there were significant differences between parent and child motivation levels, the motivation remained high for both groups. DISCUSSION/ SIGNIFICANCE OF IMPACT: The data suggests a significant difference in mean child motivation and income level. Child's high motivation may be from the idea of participating in something new, a rare opportunity for low-income children. To improve the implementation and efficacy of FBT, further study into the relationship between motivation and income level should be done.

\section{Adapting CAPABLE as CAPABLE-FAMILY for the caregiver-care recipient dyad with Alzheimer's dementia}

Emerald Rivers, Johns Hopkins University ${ }^{1}$, Janiece Taylor, PhD, MSN, $\mathrm{RN}^{1}$, Erika Hornstein, MBA, MA ${ }^{1}$, and Sarah Szanton, PhD, ANP, FAAN ${ }^{1}$ Johns Hopkins University

OBJECTIVES/GOALS: The purpose of this 4-phase exploratory study is to create a human-centered protocol for a new program, CAPABLEFAMILY, to address older adults with physical disability and dementia and their caregivers. METHODS/STUDY POPULATION: The Szanton-Gill Resilience Model, Verbrugge \& Jett Disablement, and Life Span Theory of Control are theoretical frameworks guiding this study. Phase 1. Using qualitative research $(\mathrm{n}=10$ dyads) methods (ex. Photovoice) we seek to understand the context for older adults with dementia regarding disability. Phase 2. Using synthesis/ideation (n $=10$ dyads) tools (ex. Journey Maps) we will synthesize the qualitative research during group ideation sessions with stakeholders. Phase 3. Using prototype testing ( $\mathrm{n}=3$ dyads) methods (ex. Semantic Differentials, Storyboards), we will build the most promising prototypes. Phase 4. Using an open-label pilot ( $n=3$ dyads), we will test the interventions. RESULTS/ANTICIPATED RESULTS: We will assess disability (ADL, IADL), environmental changes pain, depression, polypharmacy, provider communication needs, and caregiver burden. We will interview the dyads and multiple CAPABLE Registered Nurses (RN), Occupational Therapists (OT), and Handymen (HM) about their prior experiences and perceptions of the pilot. CFQ, MocA, pain (BPI), and ZBI will be measured pre/post $\mathrm{RN}, \mathrm{OT}, \mathrm{HM}$ visit. While there are evidence-based programs to separately address cognitive impairment and physical disability, we anticipate this is the first study to develop a community-based goal-directed, human-centered approach for the maintenance of physical function for persons with dementia in the home. DISCUSSION/SIGNIFICANCE OF IMPACT: Persons with more cognitive impairment did not improve as much as those with less cognitive impairment in the original CAPABLE study, an evidenced based program which reduced disability. This suggests the need to adapt CAPABLE to reduce the burden of disability in persons with Alzheimer's dementia. CONFLICT OF INTEREST DESCRIPTION: None
An investigation into the use of curcumin, a topical herbal agent, for the treatment of cervical intraepithelial neoplasia

Emily Wang ${ }^{1}$, Theresa Kuhn², Cecile Lahiri², Minh Nguyen², Ighovwerha Ofotokun ${ }^{2}$, Rachael Abraham², Kirk Easley ${ }^{3}$, Marina Mosunjac $^{2}$, Talaat Tadros ${ }^{2}$, Catherine Finneran², and Lisa Flowers ${ }^{2}$ ${ }^{1}$ Morehouse School of Medicine; ${ }^{2}$ Emory University School of Medicine; ${ }^{3}$ Emory University Rollins School of Public Health

OBJECTIVES/GOALS: Cervical cancer is the fourth most common cancer among women worldwide, with approximately 570,000 newly diagnosed cases and 311,000 related deaths among women in 2018.In the United States, approximately 13,000 new cases of cervical cancer are diagnosed annually with approximately 4,000 women dying each year from cervical cancer. Nearly all cervical cancer is caused by oncogenic strains of the human papillomavirus (HPV). Prevention strategies to reduce cervical cancer after HPV exposure entail treatment of cervical dysplasia at the premalignant state, specifically low-grade squamous intraepithelial lesions (LSIL) and high-grade squamous intraepithelial lesions (HSIL), along with the eventual clearance of HPV.The most common treatment for persistent LSIL or HSIL is the loop electrical excisional procedure (LEEP). This procedure is unfortunately not widely available in resource-limited countries and is associated with potential significant morbidities, including decreased fertility, preterm birth, premature rupture of membranes and cervical incompetence. Despite undergoing standard of care treatment, in certain high-risk populations, specifically HIV-infected women, there is a higher rate of premalignant HPV-related cervical disease persistence, progression and recurrence.There is a paramount need for novel nonsurgical treatments to stabilize or treat precancerous lesions of the cervix and to decrease the persistence of HPV infection. Medical treatment with the natural herb curcumin may allow subjects to receive treatment of cervical lesions without undergoing a surgical procedure.Curcumin is a major active component extracted from turmeric with anti-inflammatory, anti-infectious, and anti-cancer properties.Topical intravaginal curcumin has the promise of delivering this drug directly to the site of disease, ensuring adequate concentrations at the site of disease while avoiding systemic side effects. This proposed study will determine if there are higher rates of HPV clearance after curcumin administration among women with and without HIV who have premalignant HPV-related cervical disease, specifically LSIL or recently treated (with a LEEP) HSIL. METHODS/STUDY POPULATION: We are proposing a prospective double-blind randomized control trial to investigate the utility of topical intra-vaginal curcumin in increasing rates of HPV clearance and mitigating the high rates of disease recurrence in women with and without HIV. A sample of approximately 200 women with and without HIV who have biopsy-proven LSIL or recently treated HSIL will be randomized to one of two arms: $2000 \mathrm{mg}$ of curcumin powder in capsules or placebo inserted intravaginally at bedtime once weekly for 20 weeks (excluding the time of menses). Cervical cytology and HPV testing will be performed at baseline and 6 months post-randomization. If a participant has abnormal cytology or a positive highrisk HPV test 6 months post-randomization, they will be scheduled to undergo a colposcopy with biopsies of all suspicious cervical lesions. If biopsy results are HSIL or greater, subjects will be referred back to routine clinical treatment, which may include a LEEP. Clinicians performing the colposcopies and the study participants will be blinded since the placebo has the same appearance as the curcumin capsules. At the end 